\title{
Nosocomial outbreak of multi-resistant Streptococcus pneumoniae serotype 15A in a centre for chronic pulmonary diseases
}

\author{
Guido J. H. Bastiaens ${ }^{1 *} \mathbb{D}$, Amelieke J. H. Cremers ${ }^{1}$, Jordy P. M. Coolen', Mayke T. Nillesen ${ }^{1}$, Martin J. Boeree², \\ Joost Hopman ${ }^{1+}$ and Heiman F. L. Wertheim ${ }^{1+}$
}

\begin{abstract}
We report nosocomial transmission of multi-resistant serotype 15A Streptococcus pneumoniae (MRSP) that resulted in two lower respiratory tract infections in a centre for chronic pulmonary diseases. This outbreak highlights the potential for transmission of MRSP among vulnerable patients when laboratory turnaround time is long and patient compliance with transmission-based precautions is low.
\end{abstract}

Keywords: Streptococcus pneumoniae, Cross infection, Nosocomial, Antimicrobial resistance, Whole-genome sequencing

\section{Main text}

Streptococcus pneumoniae is a major cause of community-acquired pneumonia, bacteraemia and meningitis [1]. Asymptomatic nasopharyngeal colonization is a predisposing factor for pneumococcal infection [2]. Infections arising in hospitalized patients are often regarded as a result of earlier acquisition in the community [2], although hospital outbreaks of susceptible and resistant pneumococci have been reported [3-6]. Here, we describe two risk factors for nosocomial transmission of multi-resistant Streptococcus pneumoniae (MRSP) that resulted in two cases of hospital-acquired pneumonia.

Patient A (index case) was admitted to the Radboud University Medical Center Dekkerswald, a centre for pulmonary rehabilitation, tuberculosis and lung diseases (Groesbeek, The Netherlands) because of psychosocial issues and a mild exacerbation of chronic obstructive pulmonary disease (COPD) probably due to smoking. There were no signs of infection. This patient had been a carrier of MRSP 3.5 years ago, but as MRSP had not been identified in subsequent sputum cultures the patient was regarded as MRSP negative. On day 9 after admission a

\footnotetext{
* Correspondence: Guido.Bastiaens@Radboudumc.nl

† Joost Hopman and Heiman F. L. Wertheim contributed equally to this work.

${ }^{1}$ Department of Medical Microbiology \& Radboudumc Center for Infectious

Diseases, Radboud University Medical Center, Geert Grooteplein Zuid 10,

6525, GA, Nijmegen, the Netherlands

Full list of author information is available at the end of the article
}

sputum sample collected on day 3 yielded S. pneumoniae that was intermediately susceptible to penicillin and resistant to erythromycin, tetracycline and clindamycin (Fig. 1) indicating that patient A was an asymptomatic carrier of MRSP again. Subsequently, the patient was put on droplet and contact isolation according to protocol and discharged on day 17 (Fig. 1). Patient B was admitted to an adjacent room on the same day as patient A because of an exacerbation of COPD. Patient B was discharged after 6 days; readmitted three days later (on day 10) with a hospital acquired pneumonia, and placed on contact precautions on day 11 because of a rhino- or enterovirus positive throat swab. On day 17 (day 7 after second admission) S. pneumoniae with an antibiotic susceptibility pattern similar to that of patient A was isolated from a sputum sample collected on admission. Extension to droplet and contact isolation for MRSP was, however, not installed. Patient B was discharged on day 20. On day 27 patient $C$ was admitted with an exacerbation of COPD and that same day patient $\mathrm{A}$ was readmitted with recommended isolation procedures but the patient's adherence to the instructions for transmission-based precautions was poor. The patients were placed in adjacent rooms. On day 32 patient $C$ was diagnosed with a hospital acquired pneumonia and $S$. pneumoniae with an identical susceptibility profile to that of patient A was isolated from patient C's sputum, three days later. Following isolation of MRSP, patient C 


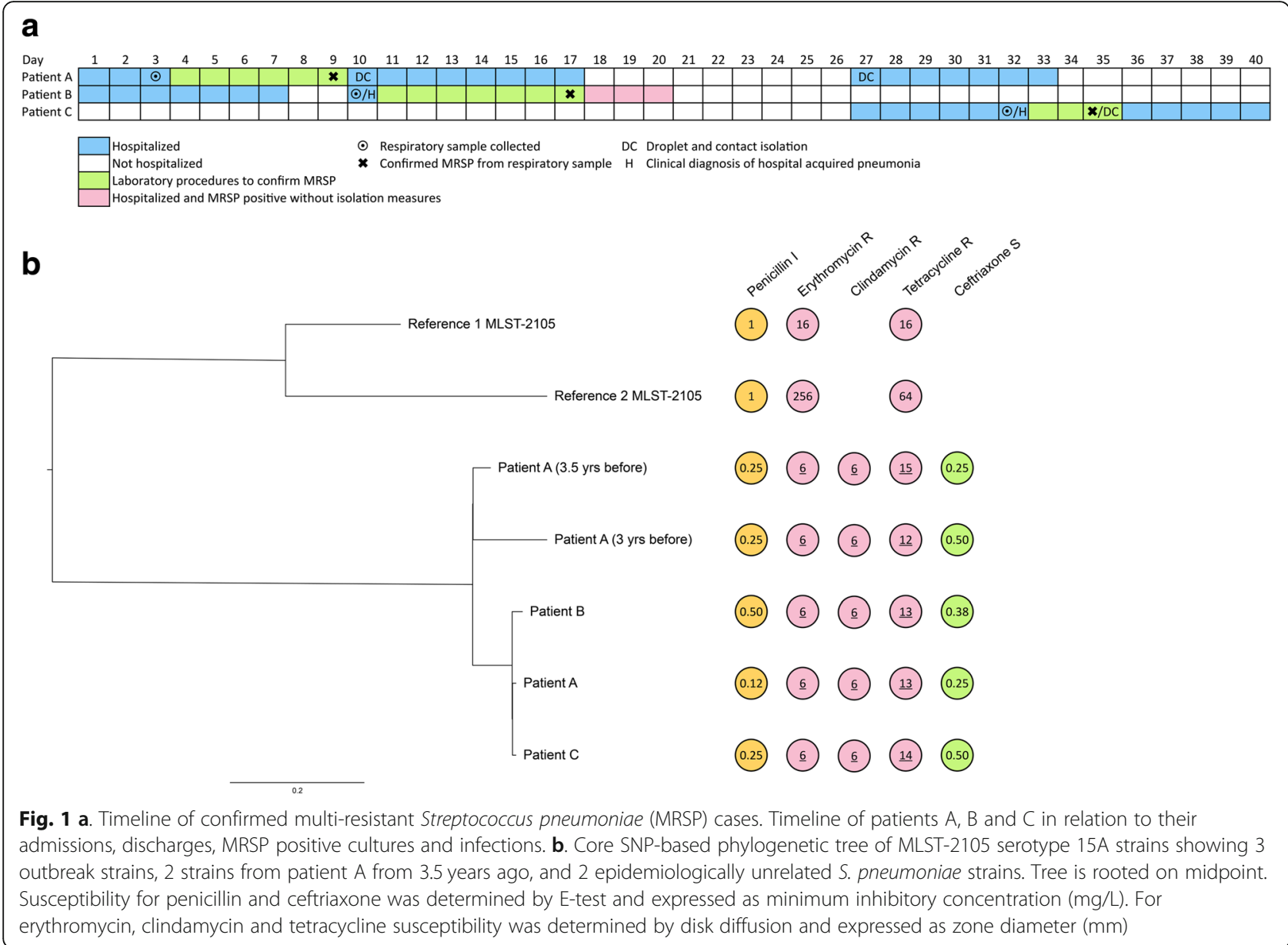

was immediately put in adequate isolation and discharged on day 40.

We suspected nosocomial transmission, since multiple S. pneumoniae, isolated from one department, showed identical but atypical colony morphology (non-mucoid, small, greyish colonies) alongside unusual susceptibility profiles (Fig. 1). In the Nijmegen area, between 2000 and 2017 not one invasive pneumococcal disease isolate had displayed concurrent reduced susceptibility to $\beta$-lactams, macrolides, lincosamides, and tetracyclines. Our suspicion for relatedness was supported by their consistent serotype $15 \mathrm{~A}$ that was assessed by Quellung reaction using Pneumococcus Neufeld Antisera (Statens Serum Institut, Copenhagen, Denmark) according to manufacturer's instructions. To rule out further transmission, we screened patients admitted to the same ward as the MRSP-positive patients and healthcare workers (HCWs) who had contact with them for carriage of MRSP using throat swabs. None of the 14 inpatients, or $78 \mathrm{HCWs}$ screened, carried MRSP, and no further cases of MRSP arose. The importance of hygiene precautions including hand hygiene was re-emphasized. Since 2014 hand hygiene compliance is monitored in Radboudumc Dekkerswald by direct observations according to the 5 moments of the World Health Organization [7]. At the time of the outbreak hand hygiene compliance was $86-93 \%$.

Nosocomial transmission of MRSP was confirmed by whole-genome sequencing (WGS) on day 41 (Fig. 1) showing that each of the outbreak isolates belonged to the rare multi locus sequence type (MLST) 2105 and carried the following antibiotic resistance genes: tet $M$ (resistance to tetracyclines) and $\operatorname{ermB}$ (resistance to macrolides and lincosamides) [8]. Based on core single nucleotide polymorphism (SNP) analysis, all three strains were highly similar and across their $>2$ million base pair long genome we detected only 4 SNPs difference at most. Compared to 2 epidemiologically unrelated serotype 15A MLST-2105 pneumococcal isolates, required for outbreak analysis given the large genetic diversity within the $S$. pneumoniae species [9], the 3 outbreak strains clustered together (Fig. 1) and were, on average, 137 SNPs different from the unrelated MLST-2105 strains. This supports the hypothesis that these cases arose from a common source.

The source of infection was presumably patient A. Although community acquisition of this MRSP by 
patients $\mathrm{B}$ and $\mathrm{C}$ cannot be fully ruled out, multiple arguments support nosocomial acquisition. The pneumococcal strain concerned is rarely encountered worldwide and is here proven to be communicable by WGS. Furthermore, patients $\mathrm{B}$ and $\mathrm{C}$ have been directly exposed to air droplets from patient $\mathrm{A}$ during regular chats, while no common source or link outside the hospital could be identified (patients were unrelated and living in different areas). Possible transmission via HCWs was investigated, yet no MRSP carriage could be identified among them, and high hand hygiene compliance (directly related to pneumococcal transmission [10]) suggested appropriate attention for hygiene precautions. Transmission to patient B probably occurred while patient $\mathrm{A}$ was not immediately isolated due to time needed for bacterial culture and susceptibility testing (Fig. 1). Patient $C$ was probably infected because patient A was not adhering to droplet and contact precautions. WGS supported our suspicion that patient A was the index case, whose MRSP isolated 3.5 years ago showed 8 SNPs difference from the current isolate, compared to 2 SNPs difference between the isolates from the 3 patients involved in this outbreak.

This outbreak highlights two risk factors for nosocomial transmission of MRSP causing hospital-acquired infections (patients B and C). First, time needed for laboratory diagnosis in low-endemic regions may lead to failure to timely install adequate transmission-based precautions. In this case the delay was due to the atypical colony morphology and resistance pattern necessitating confirmatory tests. Secondly, patient compliance with transmission-based precautions may be low despite standard instructions and may require day-to-day audits.

Considering the fact that patient A still turned out to be a MRSP-carrier after 3.5 years and recent evidence suggests that S. pneumoniae serotype and drug-resistance are associated with carriage duration [11], additional measures to prevent nosocomial spread in low-endemic regions could include routine screening at admission for patients who were colonized with MRSP previously, and placing them on droplet and contact precautions until carriage of MRSP has been ruled out.

\section{Abbreviations \\ COPD: Chronic obstructive pulmonary disease; HCWs: Healthcare workers; MLST: Multi locus sequence type.; MRSP: Multi-resistant Streptococcus pneumoniae; SNP: Single nucleotide polymorphism; WGS: Whole-genome sequencing}

\section{Acknowledgements}

We would like to thank dr. Jakko van Ingen (Department of Medical Microbiology at Radboudumc) for his help during the process of wholegenome sequencing. We also thank dr. Mark van der Linden at the German National Reference Centre for Streptococci for providing us the two MLST2105 reference strains.

Funding

Not applicable.

\section{Availability of data and materials}

All data generated or analysed during this study are included in this published article. In addition, raw sequence reads are stored in the SRA database of NCBI under following accession numbers: SAMN09812500, SAMN09812501, SAMN09812502, SAMN09812503, SAMN09812504, SAMN09812505, SAMN09812506, and part of BioProject: 485675.

\section{Authors' contributions}

GJHB, MTN, MJB, JH and HFLW were involved in management of the outbreak. GJHB, MTN, AJHC and JPMC are responsible for clinical, laboratory and WGS data collection and analysis. GJHB drafted the manuscript while critical revision was provided by AJHC, JPMC, JH and HFLW. All authors read and approved the final version of the manuscript.

\section{Ethics approval and consent to participate}

The research ethics committee of the Radboudumc judged that the study does not fall within the remit of the Medical Research Involving Human Subjects Act (WMO). The study has been reviewed by the research ethics committee on the basis of the Dutch Code of conduct for health research, the Dutch Code of conduct for responsible use, the Dutch Personal Data Protection Act and the Medical Treatment Agreement Act. The ethics committee has passed a positive judgment on the study.

\section{Consent for publication}

Not applicable.

\section{Competing interests}

The authors declare that they have no competing interests.

\section{Publisher's Note}

Springer Nature remains neutral with regard to jurisdictional claims in published maps and institutional affiliations.

\section{Author details}

'Department of Medical Microbiology \& Radboudumc Center for Infectious Diseases, Radboud University Medical Center, Geert Grooteplein Zuid 10,

6525, GA, Nijmegen, the Netherlands. ${ }^{2}$ Center for Pulmonary Rehabilitation, Radboud University Medical Center Dekkerswald, Nijmeegsebaan 31, 6561 $\mathrm{KE}$, Groesbeek, the Netherlands.

Received: 22 October 2018 Accepted: 17 December 2018

Published online: 27 December 2018

\section{References}

1. Koedel U, Scheld WM, Pfister HW. Pathogenesis and pathophysiology of pneumococcal meningitis. Lancet Infect Dis. 2002;2:721-36.

2. Bogaert D, De Groot R, Hermans PWM. Streptococcus pneumoniae colonisation: the key to pneumococcal disease. Lancet Infect Dis. 2004;4:144-54.

3. Gould FK, Magee JG, Ingham HR. A hospital outbreak of antibiotic-resistant Streptococcus pneumoniae. J Inf Secur. 1987;15:77-9.

4. Moore EP, Williams EW. Hospital transmission of multiply antibiotic-resistant Streptococcus pneumoniae. J Inf Secur. 1988;16:199-200.

5. Millar MR, Brown NM, Tobin GW, Murphy PJ, Windsor ACM, Speller DCE. Outbreak of infection with penicillin-resistant Streptococcus pneumoniae in a hospital for the elderly. J Hosp Infect. 1994;27:99-104.

6. Jauneikaite E, Khan-Orakzai Z, Kapatai G, Bloch S, Singleton J, Atkin S, et al. Nosocomial outbreak of drug-resistant streptococcus pneumoniae serotype $9 \mathrm{~V}$ in an adult respiratory medicine ward. J Clin Microbiol. 2017;55:776-82.

7. Sax H, Allegranzi B, Uçkay I, Larson E, Boyce J, Pittet D. "My five moments for hand hygiene": a user-centred design approach to understand, train, monitor and report hand hygiene. J Hosp Infect. 2007;67:9-21.

8. Kim L, McGee L, Tomczyk S, Beall B. Biological and epidemiological features of antibiotic-resistant Streptococcus pneumoniae in pre- and postconjugate vaccine eras: a United States perspective. Clin Microbiol Rev. 2016;29:525-52.

9. Cremers AJH, Mobegi FM, De Jonge MI, Van Hijum SAFT, Meis JF, Hermans PWM, et al. The post-vaccine microevolution of invasive Streptococcus pneumoniae. Sci Rep. 2015;5:14952. 
10. Connor V, German E, Pojar S, Mitsi E, Hales C, Nikolaou E, et al. Hands are vehicles for transmission of Streptococcus pneumoniae in novel controlled human infection study. Eur Respir J. 2018;52:1800599.

11. Lees JA, Croucher NJ, Goldblatt D, Nosten F, Parkhill J, Turner C, et al. Genome-wide identification of lineage and locus specific variation associated with pneumococcal carriage duration. elife. 2017;6:e26255.

Ready to submit your research? Choose BMC and benefit from:

- fast, convenient online submission

- thorough peer review by experienced researchers in your field

- rapid publication on acceptance

- support for research data, including large and complex data types

- gold Open Access which fosters wider collaboration and increased citations

- maximum visibility for your research: over $100 \mathrm{M}$ website views per year

At $B M C$, research is always in progress.

Learn more biomedcentral.com/submissions 